\title{
Oral Malodour and its Management: A Periodontal Perspective
}

\author{
Kalwa Pavankumar
}

\section{ABSTRACT}

Halitosis is a very common condition which may affect up to 30\% of the population. In most cases the aetiology of the condition is from local oral causes (oral malodour). Oral malodour is the result of the action of anaerobic bacteria in producing a range of malodorous molecular species including volatile sulphur compounds. Patients with halitosis may seek treatment from dental clinicians for their perceived oral malodour. Physiologic halitosis, oral pathologic halitosis and pseudohalitosis would be in the treatment realm of dental practitioners. Management of oral malodour is directed at managing and reducing the bacterial load both in periodontitis and in tongue coatings by instituting proper oral hygiene measures, control of tongue flora by brushing or scraping, and possibly the adjunctive use of antiseptic agents. Treatments have also been proposed to neutralise malodorous compounds by chemical agents to mask the presence of the condition. Further evidence is required to demonstrate the long-term efficacy of therapies for this troublesome condition. The purpose of this article is to review the etiology, diagnosis and treatment of oral malodor from a periodontal perspective. The review will be limited to bad breath odors originating within the mouth.

\section{Contact Author}

Dr. Kalwa Pavankumar

E-mail : drpavankumarmds@gmail.com

Key words: Halitosis, Malodour, Bad breath, Volatile sulfur compounds, Portable monitor.

$\mathrm{O}$ ral malodour (halitosis or breath odour; from the Latin for breath, Halitus), is a fairly common complaint that affects personal relationships and quality of life. Though the true prevalence is unclear, up to $30 \%$ of adults over 60 years of age report either being conscious of their oral malodour, or having been told by others they have it. In most cases, oral malodour is related to tongue microbial metabolism of local debris.

Oral malodor $(\mathrm{OM})$ has been recognized in the literature since ancient times, but in the last five to six years it has increasingly come to the forefront of public and dental professional awareness. ${ }^{1}$ Approximately $40-50 \%$ of dentists see 6-7 selfproclaimed oral malodor patients per week. ${ }^{2}$ Standard diagnosis and treatment for oral malodor in the routine care of each patient has not been established in the dental or the medical field. However the transfer of knowledge is increasing because of pioneering researchers and clinicians that have developed reputable clinics dealing with this condition. Dental and medical schools must incorporate diagnosis and treatment of oral malodor in their curriculum, so that the future generations of clinicians can effectively treat this condition.

Although this area of research has been ridiculed, at least 50\% of the population suffers from a chronic oral malodor condition by which individuals experience personal discomfort and social embarrassment leading to emotional distress. The consequences of oral malodor may be more than social; it may reflect serious local or systemic conditions. Oral Malodor research has gained momentum with increasing suspicions being directed at the sulfur-producing bacteria as the primary source of this condition.

\section{Oral and non-oral causes}

Oral malodor can be caused by many localized and systemic disorders. Oral Malodor (OM) caused by normal physiological processes and behaviors is usually transitory. Non pathologic OM may due to hunger, low levels of salivation during sleep 
food debris, prescription drugs and smoking. ${ }^{3}$ Chronic or pathological halitosis stems from oral or non- oral sources. In addition there appear to be several other metabolic conditions involving enzymatic and transport anomalies (such as Trimethylaminuria) which lead to systemic production of volatile malodors that manifest themselves as halitosis and/or altered chemoreception. ${ }^{4}$ Some of the oral causes are periodontal disease, gingivitis, and plaque coating on the dorsum of the tongue (Fig. 1). OM may be aggravated by a reduction in salivary flow. Radiation therapy, Sjorrgen's Syndrome, some lung conditions, including cancer, peritonsillar abscess, cancer of the pharynx and cryptic tonsils can also contribute to OM. ${ }^{5}$ Nasal problems such as postnasal drip that falls at the posterior dorsum of the tongue may exacerbate the oral malodor condition. Odor generated in this manner can be easily distinguished from mouth odor by comparing the odor exiting the mouth or nose. ${ }^{6}$ The non-oral causes of OM include diabetic ketosis, uremia, gastrointestinal conditions, and irregular bowel movement, hepatic and renal failure and certain types of carcinomas such as leukemia. The accurate clinical labeling and interpretation of different oral malodors both contribute to the diagnosis and treatment of underlying disease. ${ }^{7}$ Taste and smell can be altered due to facial injuries, cosmetic surgery radiation and olfactory epithilium located on the dorsal aspect of the nose. ${ }^{8} \mathrm{~A}$ relationship between gastrointestinal diseases such as gastritis and oral malodor has not been established. However, oral malodor has been reported in some patients with a history of gastritis, or duodenal and gastric ulcers. ${ }^{9}$

Saliva plays a central role in the formation of oral malodor. Such formation has its basis due to bacterial putrefaction, the degradation of proteins, and the resulting amino acids produced by microorganisms. ${ }^{10}$ Many patients with a chief complaint of oral malodor have some level of gingival and or periodontal pathology sufficient to be the etiology, but clearly periodontal pathology is not a prerequisite for production of oral malodor. ${ }^{11}$ Medications such as antimicrobial agents,

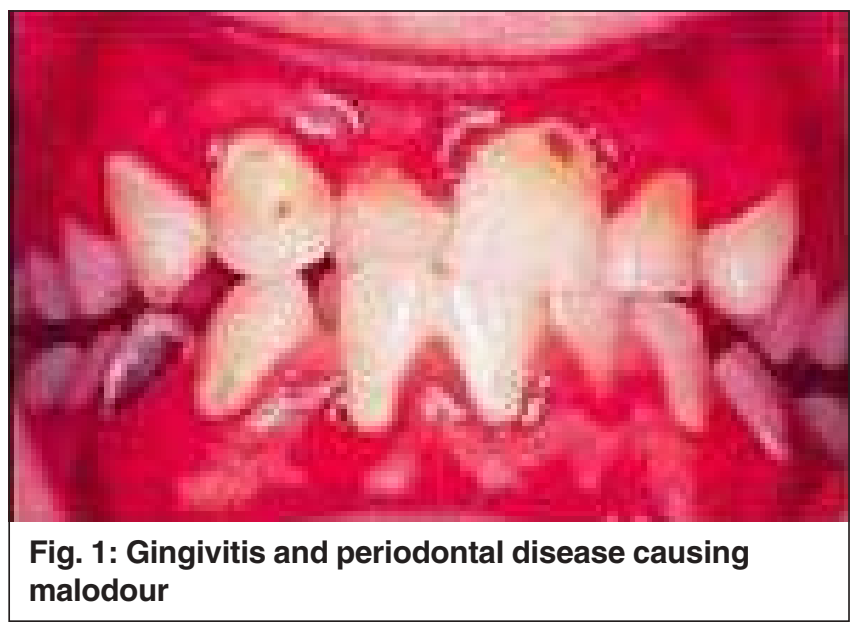

antirheumatic, anti hypertentive, antidepressants and analgesics may cause altered taste and xerostomia.

$\mathrm{OM}$ in healthy patients arises from the oral cavity and generally originates on the tongue, dorsum., ${ }^{5-12}$ The sulfur producing anaerobic bacteria appears to be the primary source of this odors. ${ }^{16}$ The large surface area of the tongue and its papillary structure allow it to retain food and debris. This is an excellent putrefactive habitat for gram negative anaerobes that metabolize proteins as an energy source. The bacteria hydrolyze the proteins to amino acids, three of which contain sulfur functional groups and are the precursors to volatile sulfur compounds (VSC's). These gaseous substances, responsible for malodor, consist primarily of hydrogen sulfide $\left(\mathrm{H}_{2} \mathrm{~S}\right)$, dimethyl sulfide $\left[\left(\mathrm{CH}_{3}\right)_{2} \mathrm{~S}\right]$, methyl mercaptan $\left(\mathrm{CH}_{3} \mathrm{SH}\right)$ and sulfur dioxide $\left(\mathrm{SO}_{2}\right) \cdot{ }^{10,12,14,15}$ Cadaverine levels have been reported to be associated with oral malodor and this association may be independent of VSC. ${ }^{17}$ Subjects challenged with cysteine rinses produced high oral concentrations of VSC, which thus seems to be a major substrate for VSC production. The other sulfur-containing substrates had much less effect. It was found that the tongue was the major site for VSC production. ${ }^{18}$

\section{The tongue plaque coating}

Research suggests that the tongue is the primary site in the production of OM. The dorsoposterior surface of the tongue has been identified as the principal location for the intraoral generation of VSC's. ${ }^{19}$ The tongue is a haven for the growth of microorganisms since the papillary nature of the tongue dorsum creates a unique ecological site that provides an extremely large surface area, favoring the accumulation of oral bacteria. The proteolytic, anaerobic bacteria that reside on the tongue play an essential part in the development of oral malodor. The presence of tongue coating has been shown to have a correlation with the density or total number of bacteria in the tongue plaque coating. ${ }^{20}$ The weight of the tongue coating in periodontal patients was elevated to $90 \mathrm{mg}$, while the VSC was increased by a factor of four. The $\mathrm{CH}_{3} \mathrm{SH} / \mathrm{H}_{2} \mathrm{~S}$ fraction was increased 30-fold when compared with individuals with healthy periodontium. This high ratio of amino acids can be due to free amino acids in the cervicular fluid when compared with those of L-cysteine. ${ }^{19}$ The BANA (Benzoyl-DL-arginine-2 napthylamide) test has been used to detect T.denticola and P.gingivalis. The two organisms that may contribute to oral malodor can be easily detected by their capacity to hydrolyze BANA a trypsin-like substrate. BANA scores are associated with a component of oral malodor, which is independent of volatile sulfide measurements, and suggest its use as an adjunct test to volatile sulfide measurement. ${ }^{21}$ Higher mouth odor organoleptic scores are associated with heavy tongue coating and correlate with the bacterial density on the tongue and it also correlates to BANA-hydrolyzing bacteria - T.denticola, P.gingivalis, and Bacteroides forsythus ${ }^{22}$ (Fig. 2). 


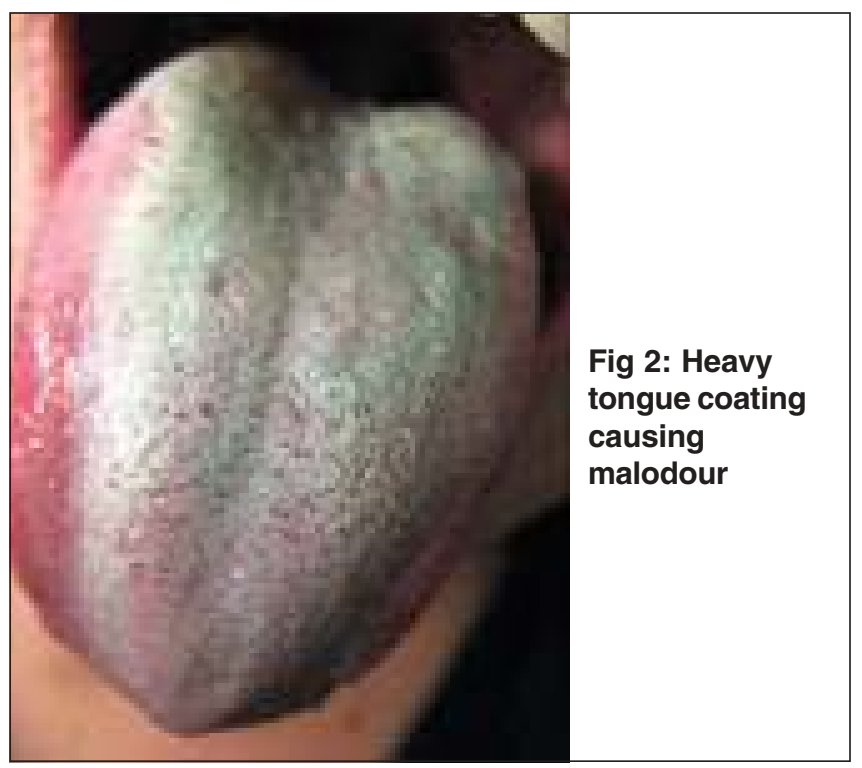

\section{Microbiota associated with oral malodor}

The actual bacterial species that cause OM have yet to be identified from among the 300 plus bacterial species in the mouth. Putrefaction is thought to occur under anaerobic conditions, involving a range of gram-negative bacteria such as Fusobacterium, Veillonella, T.denticola, P. gingivalis, Bacteroides and Peptostreptococcus. ${ }^{22,23}$ Studies have shown that essentially all odor production is a result of gram-negative bacterial metabolism and that the gram-positive bacteria contribute very little odor. ${ }^{24}$ Fusobacterium nucleatum is one of the predominant organisms associated with gingivitis and periodontitis and this organism produces high levels of VSC's. The nutrients for the bacteria are provided by oral fluids, tissue and food debris. Methionine is reduced to methyl mercaptan and cysteine. Cysteine is reduced to cystine, which is further reduced to hydrogen sulfide in the presence of sulfhydrasepositive microbes. This activity is favored at a $\mathrm{pH}$ of 7.2 and inhibited at a $\mathrm{pH}$ of $6.5 .^{10,12,14,15}$ Isolates of Klebsiella and Enterobacter emitted foul odors in vitro which resembled bad breath with concomitant production of volatile sulfides and Cadaverine both compounds related to bad breath in denture wearers. ${ }^{25}$ The amounts of volatile sulfur compounds (VSC) and methyl mercaptan/hydrogen sulfide ratio in mouth air from patients with periodontal involvement were reported to be eight times greater than those of control subjects..$^{15}$

\section{Diagnosis}

The clinical assessment of oral malodour is usually subjective and is based on smelling the exhaled air of the mouth and nose and comparing the two (organoleptic assessment). Odour detectable from the mouth but not from the nose is likely to be of oral or pharyngeal origin. Odour from the nose alone is likely to be coming from the nose or sinuses. ${ }^{26}$ In rare instances when the odour from the nose and mouth are of similar intensity, a systemic cause of the malodour may be likely

\section{Clinical assessment of oral malodour \\ Subjective}

- Intensity: the organoleptic method

- Smelling expelled air of mouth and nose separately

- Easy to do, requires no clinical training

Quality: the hedonic method

- Rarely clinically applicable

- Requires well trained clinical judges

\section{Objective}

- Detection of sulphides with appropriate monitor-simple, but may fail to detect oral malodour caused by non-sulphide components

- Gas chromatography: not applicable for routine clinical practice

- Bacterial detection (such as benzoyl-argininenaphthylamide test, polymerase chain reaction, dark field microscopy) — not applicable for routine clinical practice

Assessment of the quality of the odour (the hedonic method) relies on the use of trained clinical judges. ${ }^{27,28}$ Objective measurement of the breath components is rarely used in routine clinical practice, as it is expensive and time consuming. Volatile sulphur compounds can be measured by using a portable sulphide monitor, but as oral malodour may comprise agents other than volatile sulphur compounds this may provide an inaccurate assessment of the source and intensity of oral malodour. Gas chromatography of oral breath is a potential method of determining the components of oral malodour, but this is not of practical clinical application. Likewise, the detection of trypsin-like activities of bacteria (the benzoylarginine- naphthylamide test, dark field microscopy, and real time quantitative polymerase chain reaction detection of likely causative oral microbes) falls outside the routine clinical assessment of oral malodour.

\section{Oral malodor assessment parameters \\ Oral and Non-oral detection methods}

- Self-monitoring oral malodor tests

- Spousal and friend/confidante feedback

- Spoon Test (Fig 3)

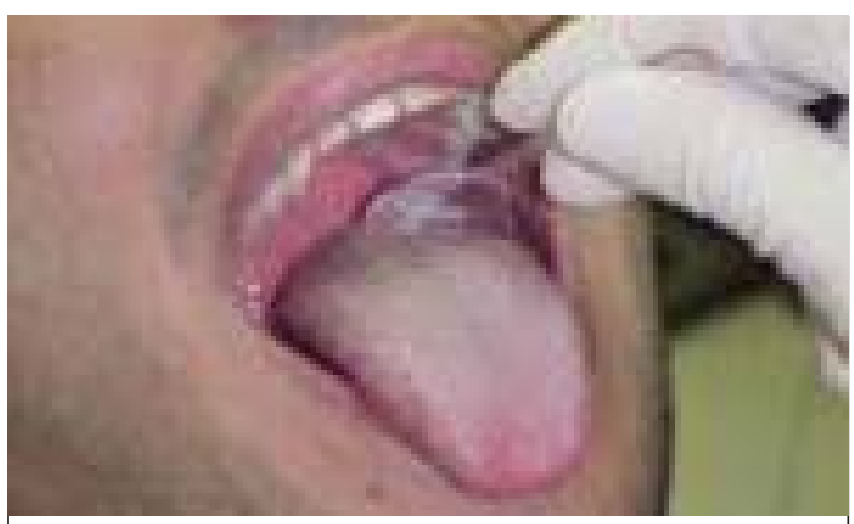

Fig. 3: Spoon test 
- Home microbial testing

- Wrist-lick test

- In-office oral malodor testing

- Odour Judges

- Microbial and fungal testing

- Salivary incubation test

- Artificial noses including the Halimeter ${ }^{\circledR}$

\section{Organoleptic measurements}

One major research problem that must be tackled is the lack of an established gold standard for rapidly measuring OM condition. The objective assessment of oral malodor is still best performed by the human sense of smell (direct sniffingorganoleptic method) but more quantifiable measures are being developed. At present, confidant feedback and expert odor (organoleptic) judges are the most commonly used approaches. Both assessments use a 0-5 scale in order to consistently quantify the odor $(0=$ No odor present, $1=$ Barely noticeable odor, $2=$ Slight but clearly noticeable odor, $3=$ Moderate odor, $4=$ Strong offensive odor, $5=$ Extremely foul odor). Individuals are instructed to refrain from using any dental products, eating or using deodorants of fragrances four hours prior to the visit to the clinic Individuals are also advised to bring their confidante or friends to assess their oral malodor.

In order to create a reproducible assessment, subjects are instructed to close their mouth for two minutes and not to swallow during that period. After two minutes the subject breathes out gently, at a distance of $10 \mathrm{~cm}$ from the nose of the counterpart and the organoleptic odors are assessed. ${ }^{29}$ In order to reduce inter-examiner variations, a panel consisting of several experienced judges is often employed. A study on the inter-examiner reproducibility indicates that there is some co-relation, albeit poor. ${ }^{30}$ Gender and age influence the performance of an organoleptic judge. Females have a better

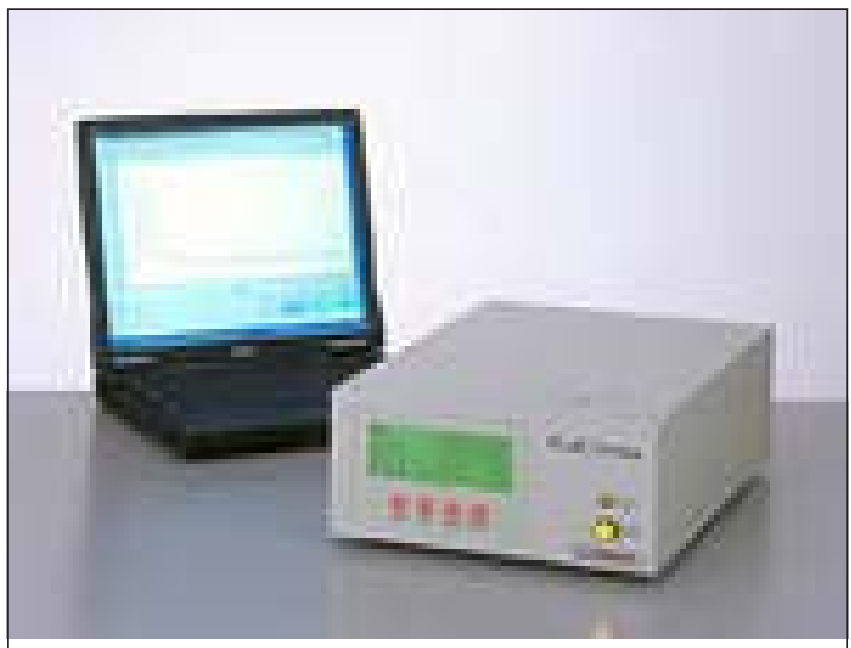

Fig. 4: Portable gas chromatograph olfactory sense and it decreases with age. Dentists and periodontists may not be ideal judges if they do not use masks on a daily basis. ${ }^{31}$

OM can be analyzed using gas chromatography (GC) coupled with flame photometric detection. ${ }^{32}$ This allows separation and quantitative measurements of the individual gasses. However the equipment necessary is expensive and requires skilled personnel to operate it. This equipment is also cumbersome and the analysis is time consuming. As a result, GC cannot be used in the dental office and is not always used in OM clinical trials (Fig. 4, 5). Recently a closed-loop trapping system followed by off-line high resolution gas chromatography ion trap detection was used for detection of compounds from saliva and tongue coating samples. ${ }^{33}$ Numerous volatile components were detected ranging from ketones to many unknowns. Adding casein (to provide cysteine and methionine) during incubation led to the appearance of nine new sulfur-containing compounds.

\section{Portable Sulfide Meter}

The portable sulfide meter (Halimeter ${ }^{\circledR}$ - Interscan Corporation, Chatsworth, CA.) has been widely used over the last few years in OM testing. The portable sulfide meter uses an electrochemical, voltametric sensor which generates a signal when it is exposed to sulfide and mercaptan gases and measures the concentration of hydrogen sulfide gas in parts per billion. The Halimeter is portable and does not require skilled personnel for operation. The main disadvantages of using this instrument are the necessity of periodic re-calibration and the measurements cannot be made in the presence of ethanol or essential oils. ${ }^{30}$ In other words, the measurements may be affected if the subject is wearing perfume, hair spray, deodorant, etc. In addition, this limitation does not allow the assessment of mouthwash efficacy until after these components have been thoroughly rinsed out or dissipated. (Fig. 6, 7).

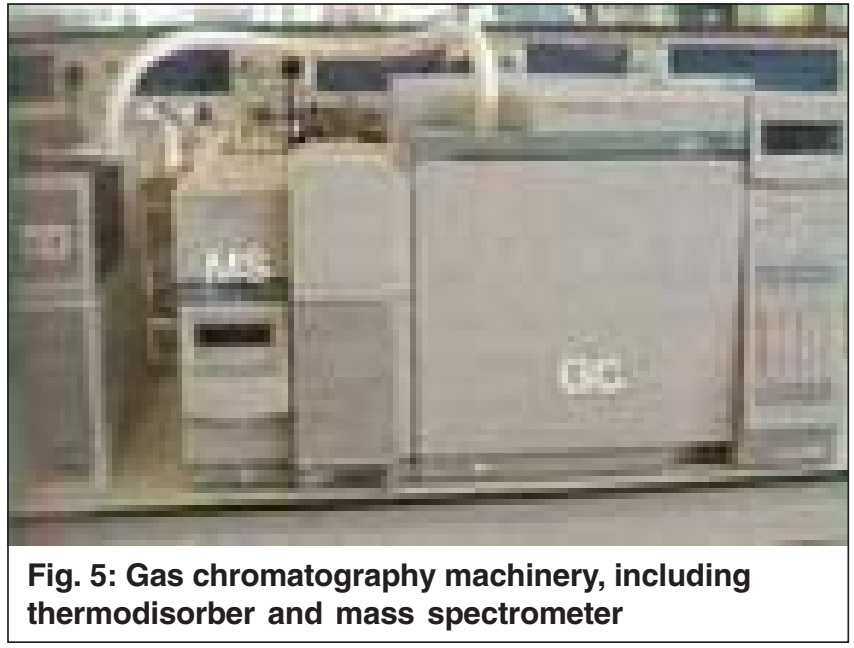




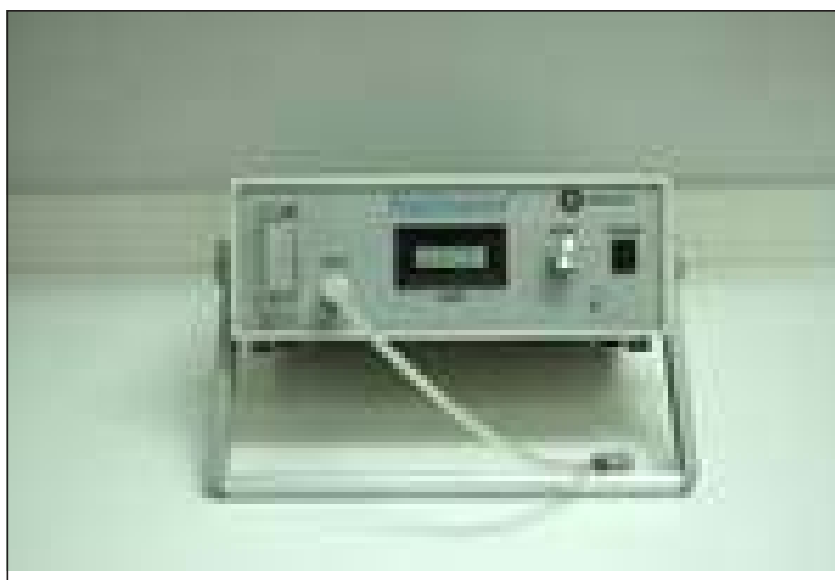

Fig. 6: Portable sulfide monitor

\section{The electronic nose}

The "Electronic Nose" is a hand held device, being developed to rapidly classify the chemicals in unidentified vapor. Its application by scientists and personnel in the medical and dental field as well as it is hoped that this technology will be inexpensive, miniaturizable and adaptable to practically any odor detecting task. ${ }^{34}$ If the Electronic Nose can learn to "smell" in a quantifiable and reproducible manner, this tool will be a revolutionary assessment technique in the field of OM. This device is based on sensor technology that can smell and produce unique fingerprints for distinct odors. Preliminary data indicates that this device has a potential to be used as a diagnostic tool to detect odors.

\section{Management of oral malodor}

Treatment is primarily directed towards educating the patient as to the cause and prevention and lessening the accumulation of oral bacteria. Effective teeth cleaning, including brushing and interdental flossing, can significantly reduce oral malodour, particularly in people with poor oral hygiene and related gingival and periodontal disease. ${ }^{35,36}$

\section{Treatment of oral malodour}

- Investigate and manage possible systemic (non-oral) source if organoleptic method detects malodour from both mouth and nose

- Improve oral hygiene by professional and patient administered tooth cleaning

- Regular atraumatic tongue cleaning

- Regular use of antimicrobial toothpastes and mouthwashes, such as

- Chlorhexidine gluconate

- Cetylpyridinium(not available in UK)

- Oil-water rinse

- Triclosan/co-polymer/sodium fluoride toothpaste

- Regular clinical review to ensure maintenance of effective oral hygiene

- Halitophobia warrants referral to clinical psychologist

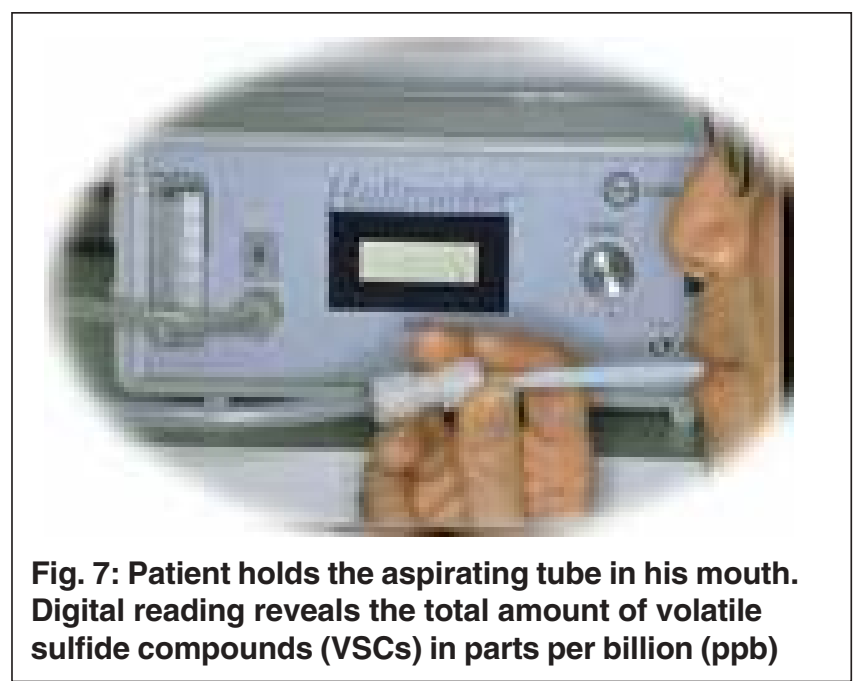

If oral hygiene is already good, or improves and yet oral malodour persists, the tongue may be the likely source of odour and hence tongue cleaning may be indicated. A recent systematic review concluded that tongue scraping may have a limited benefit in reducing oral malodour in the long term. ${ }^{37}$ Tongue scrapers facilitate the removal of bacterial accumulations in the dorsum of the tongue, which are a major cause of halitosis (Fig. 8). Nevertheless, provided tongue cleaning is done gently and regularly, no harm is likely and patients may find benefit in the short term at least. Chewing gum seems to lead to only transient reduction in oral malodour. $^{38}$

The range of mouthwashes suggested for the treatment of oral malodour act by reducing either the bacterial load or the associated odoriferous compounds. ${ }^{39}$ Unfortunately, few randomised controlled trials have looked at the effectiveness of these. Chlorhexidine gluconate produces a fall in bacteria that produce volatile sulphur compounds, and the mouthwash or spray can be more effective at reducing oral malodour for

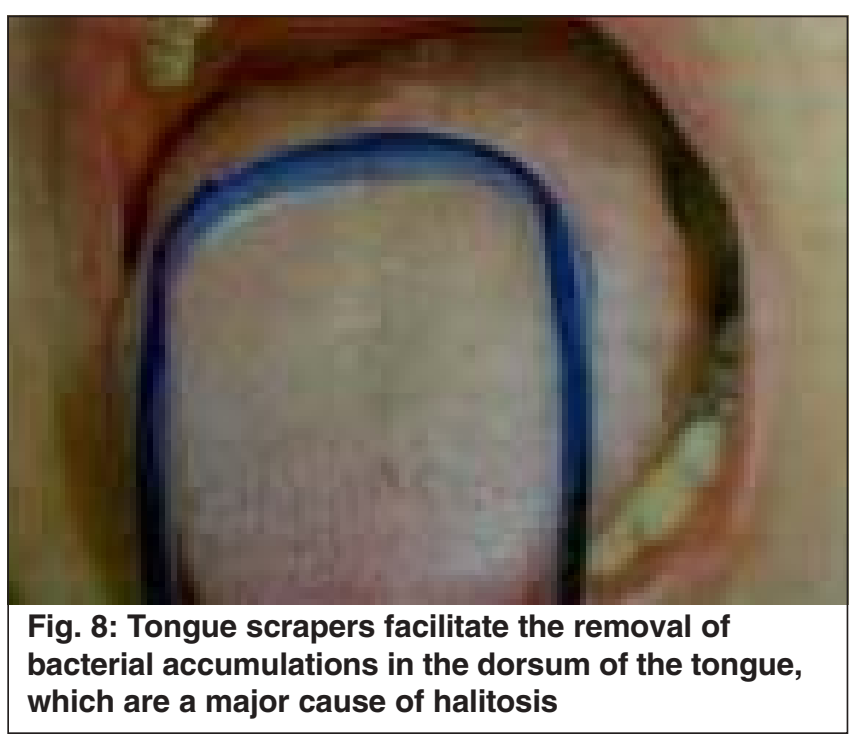


several hours than is improving oral hygiene alone. ${ }^{40} \mathrm{~A}$ mouthwash of chlorhexidine/cetylpyridinium chloride and zinc lactate also reduces oral malodour. Patients may, however, be reluctant to use chlorhexidine long term as it has an unpleasant taste, can give rise to a burning sensation of the oral mucosa if used too frequently, and can cause (reversible) staining of the teeth. A two phase oil-water mouthwash can reduce oral malodour for several hours, without adverse effects. ${ }^{41,42}$ Other mouthwashes that can reduce oral malodour for several hours include cetylpyridinium chloride, chlorine dioxide, ${ }^{43}$ and zinc chloride. ${ }^{44}$

Triclosan has both a direct action against volatile sulphur compounds and an antibacterial effect; used in mouthwashes and toothpastes, it may reduce oral malodour. The action of triclosan against volatile sulphur compounds, however, seems to depend mainly on the solubilising agent with which it is delivered. A formulation of triclosan/co-polymer/sodium fluoride seems to be particularly effective in reducing volatile sulphur compounds, oral bacteria, and oral malodour. ${ }^{45,46}$

Experimental methods of reducing oral malodour include the use of glycosylation inhibitors (such as d-galactosamine), probiotic placement of bacteria (such as Streptococcus salivarius) that replace the bacteria causing oral malodour, light exposure that directly inhibits bacteria that produce volatile sulphur compounds, ${ }^{47}$ or lethal photosensitisation.

Studies of the treatment of oral malodour have tended to report only short term data (weeks to a few months). Clearly, however, patients must continue to maintain an effective oral hygiene regimen. Patients are often unable to accurately assess the severity of their own oral odour, so that they may be unable to perceive any significant reduction in objective malodour. ${ }^{48}$ Long term support and encouragement thus needs to be provided by the patient's primary healthcare clinician, family, and friends. Certainly, those patients who are aware of their good clinical response do have an improvement in their quality of life.

Patients with halitophobia require referral for clinical psychology investigation and treatment. Unfortunately, few of these people are willing to follow this course of treatment.

\section{Discussion}

Smell and taste are interrelated in that taste or smell in isolation do not function fully if any of the receptors or innervations are blocked to either sensation - i.e., disruption of one sense compromises the function of the other. ${ }^{49}$ The olfactory nerve (first cranial nerve) for smell, and the lingual branch of the trigeminal (fifth) nerve, the corda tympanic branch of the facial (seventh), the lingual portion of the glossopharyngeal (ninth) and a branch to the palate and epiglottis from the vagus (tenth) nerve, all must be functionally intact to ensure ideal function of smell and taste..$^{50}$ The physiology, structure and function of smell and taste sensations have been comprehensively described and reviewed elsewhere. ${ }^{50}$

With oral malodor, a number of individually bad smelling substances combine to produce a stink that is unpleasant. Detecting, appreciating and describing the stink can help to define its source and establish a diagnosis. Oral malodor could therefore be a feature of some oral or non oral systemic disease. ${ }^{51}$ However, there are healthy individuals who complain of having bad breath that no one else can smell and for which no local or systemic condition can be found. Although this is sometimes referred to as "delusional halitosis," it would more accurately be described as delusional cacosmia, and is considered a monosymptomatic hypochondriacal disorder. ${ }^{52}$ A malodor is often not detected subjectively due to the phenomenon of olfactory adaptation. Approximately 80 per cent of the oral malodor that manifests as fetor oris originates from the mouth, ${ }^{53}$ and physicians should readily refer these patients to dentists for appropriate treatment and oral hygiene management.

Hydrogen sulphide $\left(\mathrm{H}_{2} \mathrm{~S}\right)$ and mercaptan $\left(\mathrm{CH}_{3} \mathrm{SH}\right)$ are most commonly associated with oral malodor, but other volatile sulphur compounds are also involved, such as dimethylsulphide $\left(\mathrm{CH}_{3}-\mathrm{S}-\mathrm{CH}_{3}\right) \cdot{ }^{54}$ The intensity of oral malodor fetor oris increases eight times or more in mouths affected by periodontal disease. This fetor oris is caused by an increase in volatile sulphur compounds and an increased mercaptan/ hydrogen sulphide ratio. ${ }^{55}$ Ketobutyrate is a product of the essential sulphur containing amino acids, namely cysteine and methionine, and is metabolized to mercaptan. Bacterial enzymes such as L-cystine desulfhydrases and 1-methionine gama-lyase break down cystine, cysteine, and methionine to produce mercaptan. ${ }^{56}$

Oral malodor is an ubiquitous and common condition. Since this is a distressing symptom for patients, the need to diagnose the underlying cause is very important. Once diagnosed, the appropriate therapy can be instituted. The cause, if possible, should be defined by the treating doctor so as to help the patient.

Management of oral malodor is therefore dependent on diagnosing the foul breath as ozostomia, stomatodysodia, physiological or pathological halitosis, or fetor oris. Therapy should then be appropriately and specifically directed to the cause of the oral malodor to

\section{Conclusion}

At least half the world's population suffers from oral malodor, and most of it originates in the mouth. The nose is the primary diagnostic tool, with optional devices that measure volatile sulfur compounds as quantitative tools to augment and quantify the process. Organized dentistry and govern-ment 
agencies can more strongly educate the public that proper oral care, including tongue cleaning, are the real "bad breath busters." Many of the mouth rinses available today are being used for the prevention and or treatment of oral malodor. Much more research is required to develop an efficacious mouth rinse for the alleviation of oral malodor. The treatment of oral malodor is relatively a new field in dentistry and many of the treatments thus far have involved a trial and error approach, but the knowledge and experience gained so far will hopefully facilitate clinical investigations in this field and eventually lead to improved diagnostic techniques and treatment products.

\section{THE AUTHORS}

\section{Dr Kalwa Pavankumar, MDS, DHM}

Assistant Professor

Department of Periodontics

Navodaya Dental College \& Hospital

Navodaya Nagar, RAICHUR-584103

Karnataka, India

\section{Corresponding address:}

Dr. Kalwa Pavankumar

S/o K.Vijay Kumar

Murali Cloth Stores, Main Road , (Post) Devarakadra

(Dist) Mahabubnagar - 509204.

Andhra Pradesh, INDIA

Mob: +91-9902617087, +91-9705645566

E-mail: drpavankumarmds@gmail.com

\section{References}

1. Nachnani S, Oral Malodor. A Brief Review CDHA Journal 1999; 14(2):13-15.

2. Survey conducted at ADA reveals interesting trends. Dent Econ 1995;6.

3. Scully C, Porter R, Greenman J. What to do about halitosis? Brit Med J 1994;308:217-18.

4. Preti G, Clark L, Cowart BJ, et al. Non oral etiologies of oral malodor and altered chemosensation. J Periodontol 1992;63(9): 790-96.

5. Young K, Oxtoby A. Field EA. Halitosis: A review. Dental Update 1993;20:57-61.

6. Rosenberg M. Clinical assessment of bad breath: current concepts. J Am Dent Assoc 1996;127(4):475-82.

7. Touyz LZ. Oral Malodor-a review. J Can Dent Assoc 1993;(7): 607-10.

8. Ship JA. Gastatory and olfactory considerations. Examination and treatment in general practice. J Am Dent Assoc 1993;31:53-73.

9. Tiomny E, Arber N, Moshkowitz M, Peled Y, Gilat T. Halitosis and Helicobacter pylori. J Clin Gastroenterl 1993;15:236-37.

10. Kleinberg I, Westbay G. Salivary metabolic factors and involved in oral malodor formation. J periodont 1992;63(9):768-75.

11. Newman MG. The Role of periodontitis in Oral Malodor: Clinical Perspectives Bad breath: A multidisciplinary approach. van Daniel Steenberghe and Mel Rosenberg Editors.

12. Rosenberg M, Editor. Bad Breath: research perspectives. Ramot Pub Tel Aviv 1995.

13. Tessier JF, Kulkarni GV. Bad Breath: etiology and treatment. Periodontics Oral Health 1991;19-24.

14. Tonzetich J. Production and origin of oral malodor: a review of mechanisms and methods of analysis. J Periodontol 1977;28: 13-20.
15. Yaegaki K, Sanada K. Biochemical and clinical factors influencing oral malodor in periodontal patients. J Periodontol 1992;63:78389

16. Clark G, Nachnani S, Messadi D, CDA Journal 1997;25(2).

17. Goldberg S, Kozlovsky A, Gordon D, Gelernter I, Sintov A, Rosenberg M. Cadaverine as a putative component of oral malodor. J Dent Res 1994;73(6):1168-72.

18. Waler $\mathrm{S}$. On the transformation of sulfur-containing amino acids and peptides to volatile sulfur compounds (VSC) in the human mouth. Eur J Oral Sci 1997;105(5 pt 2):534-37.

19. Yaegaki K, Sanada K. Volatile sulfur compounds in mouth air from clinically healthy subjects and patients with periodontal disease. J Periodont Res 1992;27:223-38.

20. De Boever EH, Loesche WJ. The tongue microflora and tongue surface characteristics contribute to oral malodor In: van Steenberge D. Rosenberg M (eds), Bad Breath: A Multidisciplinary Approach. Leuven Belgium: Leuven University Press, 1996:111121.

21. Kozlovsky A, Gordon D, Gelernter I, Loesche WJ, Rosenberg M. Correlation between BANA test and oral malodor parameters. J Dent Res 1994;73(5):1036-42.

22. De Boever EH, Loesche WJ. Assessing the contribution of anaerobic microflora of the tongue to oral malodor. JADA 1995;126:1384-93.

23. Kleinberg I, Codipilly M. The biological basis of oral malodor formation In Rosenberg M. (Ed) Bad Breath: research Perspective. Tel Aviv, Israel Ramot Publishing. Tel Aviv University 1995:13-39.

24. McNamara TF, Alexander JF, Lee M. The role of microorganisms in the production of oral malodor. Oral Surg 1972;34:41

25. Goldberg S, Kozlovsky A, Gordon D, Gelernter I, Sintov A, Rosenberg M. Cadaverine as a putative component of oral malodor. J Dent Res 1994;73(6):1168-72.

26. Rosenberg M, McCullough CA. Measurements of oral malodor: current methods and future prospects. J Periodontol 1992;63:77682.

27. Greenman J, Duffield J, Spencer P, Rosenberg M, Corry D, Saad S, et al. Study on the organleptic intensity scale for measuring oral malodor. J Dent Res 2004;83:81-5.

28. Nachnani S, Majerus G, Lenton P, Hodges J, Magallanes E. Effects of training on odor judges scoring intensity. Oral Dis 2005;11:40-4.

29. Rosenberg M, Septon I, Eli I, Bar-Ness A, Gelenter I, Bremer S, Gabbay. Halitosis measurement by an industrial sulfide monitor. $J$ Periodon 1991;62:487-89.

30. Rosenberg M, Kulkarni GV, Bosy A, McCulloch CAG. Reproducibility and sensitivity of oral malodor measurements with a portable sulfide monitor. J Dent Res 1991;11:1436-40.

31. Doty RL, Green PA, Ram C, Yankel SL. Communication of gender from human breath odors: Relationship to perceived intensity and pleasantness. Norm Behav 1982;16:13-22.

32. Tonzetich J, Richter VJ. Evaluation of volatile odiferous components of saliva. Arch Oral Biol 1964;9:39-45.

33. Claus D, Geypens B, Rutgeers P, Ghyselen J, Hoshi K, van Steenberghe $\mathrm{D}$, et al. Where gastroenterology and periodontology meet: determination of oral volatile organic compounds using closed-loop trapping and high resolution gas chromatography ion trap detection. In: van Steenberghe D, Rosenberg M, Bad Breath: A multidisciplinary approach. Leuven University Press. Leuven, Belgium 1998:pg17-30.

34. Gibson TD, Prosser O, Hulbert JN, et al. Detection and simultaneous identification of microorganisms from headspace samples using an electronic nose. Sensors and Actuators 1970; B44:413-22.

35. Morita M, Wang HL. Association between oral malodour and adult periodontitis: a review. J Clin Periodontol 2001;28:813-19.

36. Rosenberg M. Clinical assessment of bad breath: current concepts. JAm Dent Assoc 1996;127:475-82. 
37. Outhouse TL, Al-Alawi R, Fedorowicz Z, Keenan JV. Tongue scraping for treating halitosis. Cochrane Database Syst Rev 2006;(2):CD005519.

38. Reingewirtz Y, Girault O, Reingewirtz N, Senger B, Tenenbaum $H$. Mechanical effects and volatile sulphur compound-reducing effects of chewing gums: comparison between test and base gums and a control group. Quintessence Int 1999;30:319-23.

39. Scully, C, Porter, SR. Halitosis.

40. Quirynen M, Mongardini C, van Steenberghe D. The effect of a 1-stage full-mouth disinfection on oral malodour and microbial colonization of the tongue in periodontitis: a pilot study. $J$ Periodontol 1998;69:374-82.

41. Yaegaki K, Sanada K. Effects of a two-phase oil-water mouthwash on halitosis. Clin Prev Dent 1992;14:5-9.

42. Kozlovsky A, Goldberg S, Natour I, Rogatky-Gat A, Gelernter I, Rosenberg M. Efficacy of a 2-phase oil-water mouthrinse in controlling oral malodour, gingivitis and plaque. J Periodontol 1996;67:577-82.

43. Frascella J, Gilbert RD, Fernandez P. Odor reduction potential of a chlorine dioxide mouthrinse. J Clin Dent 1998;9:39-42.

44. Tonzetich J. Oral malodour: an indicator of health status and oral cleanliness. Int Dent J 1978;28:309-19.

45. Hu D, Zhang YP, Petrone M, Volpe AR, DeVizio W, Giniger M. Clinical effectiveness of a triclosan/copolymer/sodium fluoride dentifrice in controlling oral malodour: a 3 week clinical trial. Oral Dis 2005;11:51-3.

46. Niles HP, Hunter C, Vazquez J, Williams MI, Cummins D. The clinical comparison of a triclosan/copolymer/sodium fluoride dentifrice vs a breath-freshening dentifrice in reducing breath odor overnight: a crossover study. Oral Dis 2005;11:54-56.
47. Sterer N, Feuerstein O. Effect of visible light on malodour production by mixed oral microflora. J Med Microbiol 2005;54: 1225-29.

48. Rosenberg M, Kozlovsky A, Wind Y, Mindel E. Self assessment of oral malodour 1 year following initial consultation. Halitophobia 1999;30:324-27.

49. Bradley RM. Physiology of taste receptors. Physiology of olfactory receptors. In: Basic Oral Physiology. R.M. Bradley, ed. Chicago and London, Year Book Medical Publishers. Ch 3,21-24; Ch 4,4357, 1981.

50. Jenkins GN. Sensations arising in the mouth. In: Physiology and Biochemistry of the Mouth GN. Jenkins, ed. Oxford and London. Blackwell Scientific Publications (67 References to Basic Physiology) 1978:pp.542-570.

51. Tessier JF, Kulkarni GV. Bad breath, etiology, diagnosis and treatment. Oral Health 1991;81:19-24.

52. Iwu CO, Akpata O. Delusional halitosis. Review of the literature and analysis of 32 cases. Br Dent $J$ 1990;168:294-96.

53. Tonzetitch J. Sources measurement and implication of oral malodor. J Dent Res 1991;70:337, 572.

54. Schmidt NF, Missan SR, Tarbet WJ. The correlation between organoleptic mouth-odor ratings and levels of volatile sulfur compounds. Oral Surg 1978;45:560-67.

55. Kostelc JG, Zelson PR, Preti G, et al. Quantitative differences in volatiles from healthy mouths and mouths with periodontitis. Clin Chem 1981;27:842-45.

56. Yaegaki K, Suetaka T. Fractionation of the salivary cellular elements by Percoll density gradient centrifugation and the distribution of oral malodor precursors. Shigaku 1989;77:26975 . 